\title{
DYNAMIC DEPLOYMENT OF HOMOGENEOUS SENSOR NODES USING PLANT PROPAGATION ALGORITHM WITH MAXIMUM COVERAGE
}

\author{
Ramandeep Kaur \\ Department of computer sci \& Engg \\ BGIET,Sangrur, India
}

\author{
Dr.Rajesh Kumar \\ Assistant Prof. Department of computer sci \& Engg \\ BGIET, Sangrur, India
}

\begin{abstract}
Wireless sensor network serves as a nursery for umpteen cosmic fields such as defense, security, scientific applications and environment. One of the contemporary areas of research in wireless sensor network is the area coverage. In wireless sensor network, the ethereal trait of network is measured by the area covered by the sensor nodes. The quality of service is achieved by the maximum area coverage but the quality of service is inversely proportional to vendor's cost there is a need to place the more sensor than existing. The web of issues in the network coverage includes homogeneous or heterogeneous sensor nodes, random or deterministic deployment of sensor nodes and centralized and distributed algorithms[4]. Other than that, the network coverage relies upon the deployment of sensor nodes. The sensor nodes can be placed in one of the following manners, deterministic or random. In this paper, Plant Propagation Algorithm is used to improve the coverage of network nodes and deploy the nodes in such a manner that has minimum overlapping of nodes. The paper concluded that the algorithm provides maximum area coverage with same number of nodes. At the end of this paper the results of proposed algorithm are compared with genetic algorithm which shows that the Plant Propagation algorithm takes less time to eradicate the intersection between sensor node as compared to genetic algorithm.
\end{abstract}

Keywords—deployment, intersection, maximum area coverage,Plant propagation algorithm, wireless sensor network.

\section{INTRODUCTION}

Wireless sensor networks (WSN) is a group of sensors nodes which are connected to each other for passing their data elements through the network to a specified locations in a network. Mostly the wireless sensor networks (WSN) are bi-directional and allowing control over sensor node activity. Basically the development these type of networks are motivated by the areas military applications. The WSN is a combination of "nodes" from a few to thousands of sensor nodes, where each and every node is connected to one (or sometimes more) sensors. Every node in a network has various parts like a radio transceiver (which has internal antenna or sometimes it has a connection to external antenna), a microcontroller, and also has an electronic circuit that is basically used to interface with the other sensor nodes and a battery. A sensor node may vary in size from very small to very large size. The cost of network nodes is also very variable that may be ranging from a few to thousands of dollars. The cost of network nodes depends on their complexity. The Cost and size of sensor nodes in a network arise the corresponding constraint energy, computational speed and memory. The topology of the Wireless Sensor Networks also vary from a star network (simple) to multi-hop mesh network.

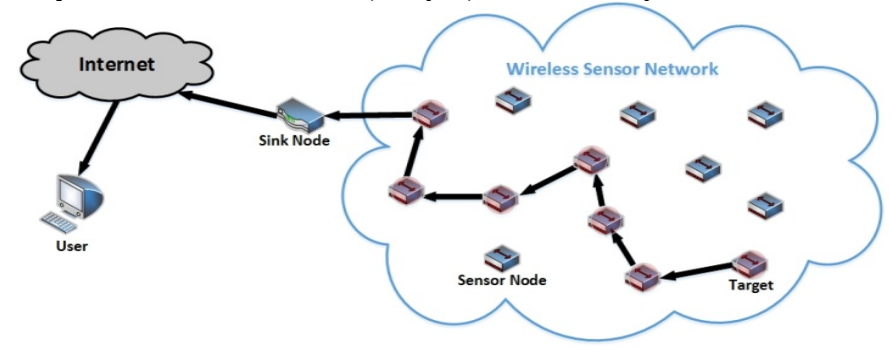

Fig.1 Wireless Sensor Network
In the recent years, wireless sensor network have its applications in every fields such as industrial sensing, habitat monitoring, infrastructure security, environment, military and traffic control to name a few. The wireless sensor network is a combination of small devices called sensor nodes. The senor nodes are operated by the battery power which is rechargeable. The wireless sensor nodes itself has the capabilities of signal processing, communication and computations. The sensor nodes communicate with each other to establish a network to monitor a geographical region. The sensor nodes also communicate through the base stations to report sensed data to each other.

in wireless sensor network the nodes are deployed either in deterministic or random fashion. In deterministic manner the nodes are placed on the predetermined locations which is very easy to design a imaginary network but it is not possible in reality to place the sensor nodes in deterministic manner[1]. On the other hand, in the random fashion the sensor nodes are dropped by plane on non-determined locations.

The main research area in wireless sensor network is a coverage. the wireless sensor network coverage is usually translated as how well the nodes will cover the given area. The coverage will help to achieve the better quality of service as well as the good transmission rate. Coverage in wireless sensor network is classified as[2]:

\section{Point coverage \\ 2. Barrier coverage \\ 3. Area coverage}

In a network of point coverage the particular points(sensor nodes) of interest are detected rather than the whole area. In this coverage strategy, target nodes can be represented as 
the set of isolated points. The main objective is to cover particular set of isolated points by least number of sensor nodes.

In the barrier coverage, boundary of a particular region is covered. Barrier coverage is particularly used in the protection areas to prevent the unwanted events. The main goal in this type of coverage is to detect intruders or any other thievery as they cross a border or as they break through a protected area.

In area coverage, the particular area of interest is covered. The area coverage has the number of sensor nodes to cover a specific area. Area coverage is used for the problem of maximization or the problem of minimization. In the maximization problem the coverage of network is increased. In the minimization problem, total area of the hole coverage is reduced.

The main active area of our research is the area coverage because the area coverage is directly or indirectly concerned with the issues in wireless sensor network. In the area coverage network the nodes that are sensed belongs to small or large network. In the small network area, the nodes are manually deployed but in the large network the nodes are deployed randomly[8]. When the nodes are randomly deployed, mostly the problem of intersection occurs which further arise the problem of high power consumption. In the survey of these problem the various algorithm proposed to maximize the area coverage and to reduce the intersection of nodes. The maximum coverage is achieved by the redeployment of nodes.

The importance of the Nature-Inspired Algorithms[3] is mainly influenced by the ability of biological systems to capably adjust to often unpredictable environment. Nature inspired technique is the computing which has its basis in the biological mechanism of the nature i.e., animals and human. The nature has four very important features which are fundamental building blocks are self healing, self optimization, self processing and self learning. Nature that work as self optimizer is that it can automatically run its resources in an well-organized manner to meet endeavor need. A strawberry plant and any other plant which always propagates through runners will do to optimize its continued existence[4]. If the plant is in a good location of the ground, with nutrients, enough water and light, then it is realistic to assume that there is no pressure on the plant to leave that location to guarantee its continued existence. So, the plant will send many short runners in all the directions that will give new plants and inhabit the neighbourhood as best they can.

\section{RELATED WORK}

Various techniques related with PPA (Plant Propagation Algorithm) have been studied for energy efficiency in wireless sensor networks[4]. In the wireless sensor network connectivity of nodes and sensing coverage area are the two basic fundamental challenges. In the literature it has been studied that when the range of particular sensor is twice of its sensing range capacity only then there is a possibility of connectivity. As related to the concept of coverage; the placement of network sensors is considered as basic and important concept. Sensors can be placed in two ways:
- Random placement

Raymond et al[1] represent a deep survey on coverage in Wireless Sensor Network. In this work the representative survey of the current given work is taken from the results which is already implemented in this area. Various terms and concepts such as types of coverage, placement of sensor nodes, node types and constraints are studied. In this paper the various issues like art gallery problem, Voronoi diagram and delaunary triangulation, worst and best case coverage, probabilistic sensing, disjoint sets and connectivity with coverage are studied. The work concluded that there exist many challenges still like basic problems related to area coverage that must be solved in future.

Anju et al [2] represent the survey on coverage problem in wireless sensor network. The author discuss the various issues in coverage and elaborated the strategies based on the Computational Geometry and other strategies. In this paper the the author tries to cover all the aspects of the problem of coverage such as deployment of nodes, various algorithms and techniques used to solve the coverage and connectivity problems. The author suggest to use the decentralized server based algorithm to solve the problems and also suggest to compare the centralized and distributed algorithm based on the factors as cost and power consumption etc

Ravneet et al [5] use Flower Pollination algorithm for selecting optimum number of Base Transceiver stations (BTS) and optimizing site locations. The survey of this paper is based on how optimally locate BTS to obtain maximum coverage with minimum infrastructure cost. In the proposed work to find the optimum number of cell sites, the base stations are located and calculate SINR, network performance and capacity. Thereafter, the flower pollination algorithm is used to find the optimum number of sites. Furthermore the proposed algorithm is compared with Artificial Bee Colony (ABC). In future the proceedings author suggest that, the various techniques can be applied to achieve full coverage in a network with minimum number of sensors.

Shubhdeep et al[6] use genetic algorithm to maximize the area, reduce the intersection of nodes and compare the results with standard genetic algorithm. The nodes are redeployed by using genetic algorithm. The processed results of genetic algorithm are compared with standard genetic algorithm and concluded that the time cost of proposed algorithm is less as compared to standard genetic algorithm. The future concern of this work is to develop the algorithm that gain the maximum energy efficiency with the maximum area coverage.

Sandeep et al[7] use the sensor deployment algorithm for the deployment of homogeneous wireless sensor network. The author discuss the problem of intersection between nodes that further arise the problem of inefficient coverage. The author concluded that the area is maximized by placing the sensor nodes in homogeneous manner with proposed algorithm. 
Prabhudutta et al[8] use the optimal deployment technique to solve the problem of energy efficiency and perform monitoring that is very efficient with less number of sensor nodes. The sensor nodes in the network are statically placed. The proposed algorithm in this work produces a particular set consisting of network nodes which would be used monitor the complexity and area consisting of target node. The proposed technique concluded that the better coverage is achieved with best life of nodes. The present performed work in this paper is implemented by using graphical representation of network nodes which can anyone to estimate the structure of number of nodes to cover a particular area. The future scope of this paper is to reduce the complexity, improve the existing algorithm or design a new algorithm is possible.

GaoJun et al[9] the survey in this paper is based on the wireless sensor network coverage. Some design consideration are also studied. The author describes the two challenges namely maximizing network life and network connectivity. The author also discuss the art gallery problem and circle covering problem. The author suggested that there must be solved the mobile sensor problem

Deying et al [10] surveys the current work of coverage problem. The author investigated two types of coverage: area coverage and target coverage. The author also studied the sensor deployment methods and wireless sensor network properties (e.g. network connectivity, fault tolerance for connectivity and energy efficiency) in detail. The author represent the comprehensive review and analysis of various existing techniques and algorithms.

S. Indhumathi* et al[11] use the gap clustering technique with genetic algorithm to solve the problem of area coverage. The author discuss the problem of overlapping which arises due to the random deployment of sensor nodes. The author analyze the type of sensor nodes calculate the coverage sensing distance, total coverage area for all the nodes in network on the radius of each node. By using the proposed algorithm, the overlapping is reduced and area is maximized.

Parul et al [12] discuss the hard real world problems and considering the aspects of these problems. The hard real world problems (NP hard and NP complete) are solved by using the nature inspired algorithms. A single platform is used to evaluate the results of maximum and minimum numerical optimization problems. The results of 12 natural algorithms are compared. The important features, time cost, input parameters are considered of these algorithms. The work provide the base for all nature inspired computational problems.

\section{A.Research Objectives}

\section{PROPOSED WORK}

1) Study existing algorithm

2) Apply the proposed algorithm.

3) Simulate and validate the results.

4) Result analysis.

\section{B.Methodology}

In the proposed work the dynamic deployment of homogeneous nodes is done by using Plant propagation Algorithm. The dynamic deployment of sensor nodes is the re-structuring or re-deployment of network nodes, sink node and happening is possible. The main area deals with the portability of network nodes. The sensor nodes are repositioned to gain the maximum coverage of the area by network nodes. Initially the deployment of nodes is random that is the nodes are not placed according to any specified manner. The intersection of between sensor nodes is calculated by proposed formula. If any network node has an overlapped area then that problem is eliminated by the method of Plant Propagation Algorithm. Before further processing to the method description, the demonstration of the sensor nodes with non-intersection and network nodes with intersection have been reviewed. The following figure shows and concept of intersection.

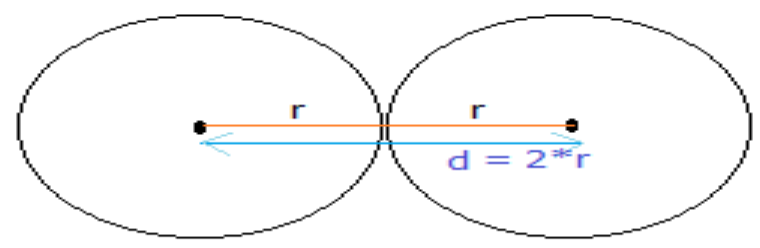

Fig. 2. Network nodes with no intersection area

In the above figure ' $d$ ' represent the fitness function that shows no overlapping and ' $r$ ' represents the range of nodes



Fig. 3. Distance ' $\mathrm{D}$ ' between two nodes having overlapping area

In the above figure ' $\mathrm{D}$ ' represent the distance between center of network nodes when intersection exists. D varies with different values according to area.

When the overlapping is found between the nodes the Plant Propagation algorithm is applied to the problem specific area. The new runners are generated from the particular problem prone sensor nodes. The runners are generated by using a number of iterations until the overlapping will be removed. When a runner is generated the following process is used:

the range of the node is calculated first. The range is used to specify the maximum area in which the particular sensor nodes can exist after the iteration are completed. The range of the sensor node is calculated by using the following formula:

$$
D x_{j}^{i}=2\left(1-N_{i}\right)(-0.5) \text { for } j=i \ldots . . n \text {; }
$$

After the maximum area is calculated. The $\mathrm{x}$ coordinates of the node is created. the following given equation is used to generate the population which is randomly placed. Basically this is used for $x$ coordinates:

$$
X_{i, j}=a_{j}-\left(b_{j}-a_{j}\right) \quad \text {, for } j=1 \ldots \ldots n \text {; }
$$

At the last step when $d x_{i}^{j}$ is calculated, the extent to which the generated runner will reach, the search equation for $y$ coordinates fing the next neighbourhood which is calculated as follows:

$$
Y_{i, j}=x_{i j}+\left(b_{j}-a_{j}\right) d_{i j} \text { for } j=1 \ldots . . n
$$




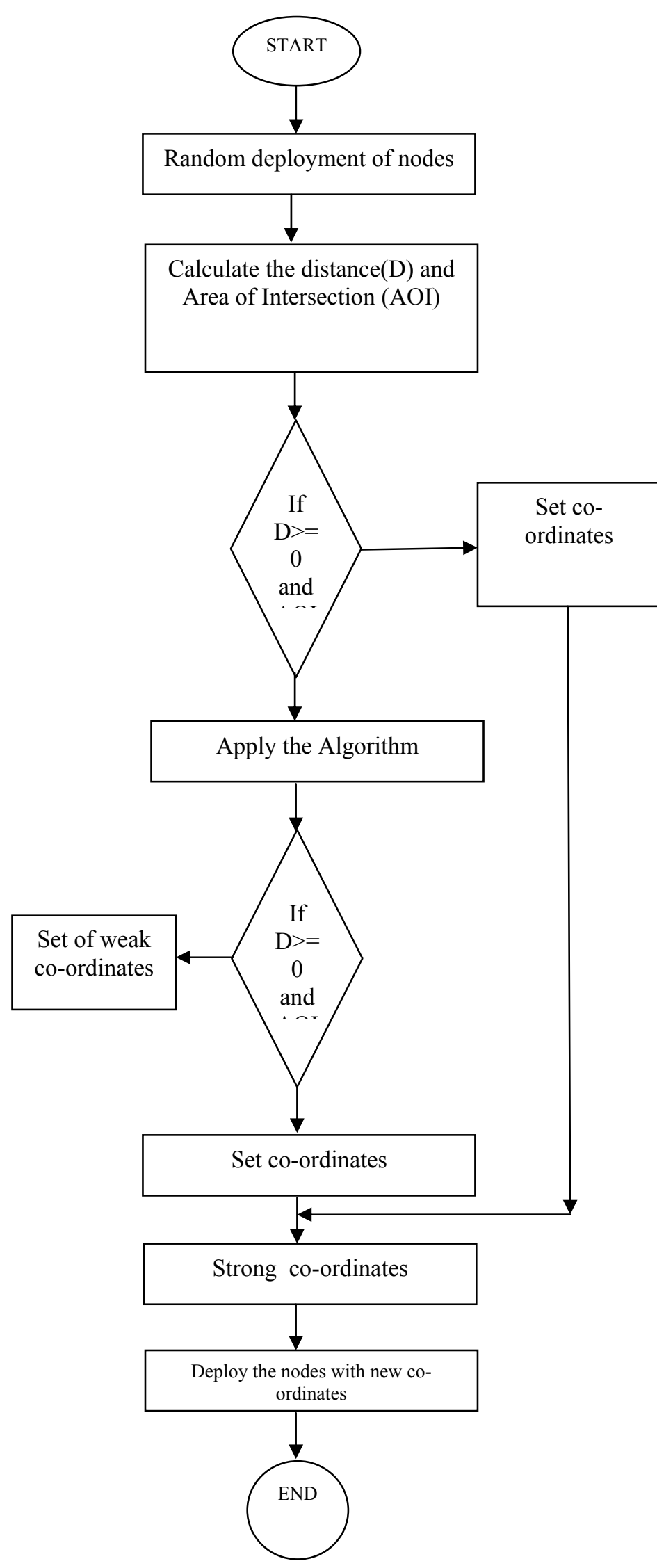

\section{PLANT PROPAGATION ALGORITHM}

A. Initial Population

Initially the population of sensor node is selected randomly. The given points $\mathrm{x}$ and $\mathrm{y}$ are the coordinate values and the value of ' $r$ ' shows the range of network nodes.
TABLE I. INITIAL FIRST POPULATION

\begin{tabular}{|c|c|c|}
\hline $\mathrm{R}$ & $\mathrm{X}$ & $\mathrm{Y}$ \\
\hline 10 & 1 & 2 \\
\hline 10 & 3 & 4 \\
\hline 10 & 4 & 3 \\
\hline 10 & 5 & 6 \\
\hline 10 & 6 & 5 \\
\hline
\end{tabular}

Step1: Every solution in the area $\mathrm{X}_{\mathrm{i}}$ generates the runner and length of the runner is inversely proportional to its growth. The following equation is used to calculate the maximum length of the runner:

$$
D x_{j}^{i}=2\left(1-N_{i}\right)(-0.5) \text { for } j=i \ldots . . n \text {; }
$$

Step2: the following given equation is used to generate the population which is randomly placed. Basically this is used for $\mathrm{x}$ coordinates:

$$
X_{i, j}=a_{j}-\left(b_{j}-a_{j}\right) \quad, \text { for } j=1 \ldots . . n \text {; }
$$

Step3: When $\mathrm{dx}_{\mathrm{i}}^{\mathrm{j}}$ is calculated, the extent to which the generated runner will reach, the search equation for $y$ coordinates fing the next neighbourhood which is calculated as follows:

$$
Y_{i, j}=x_{i j}+\left(b_{j}-a_{j}\right) d_{i j} \text { for } j=1 \ldots . n
$$

\section{B. Fitness Function}

In maximum area coverage it has been decided the coordinates of network nodes where the area under coverage is maximum and area under intersection is minimum. If the distance between randomly selected network nodes is more than or equal to ' $d$ ' (where $d=2 * r$ ) then it is said that there is no intersection and if the calculated distance is less that' $d$ ' then there is intersection. calculate the fitness function as follows:

$$
\mathrm{d}>2 \mathrm{r} \text { where } \mathrm{d}=\sqrt{ }\left(\mathrm{x}_{\mathrm{i}-\mathrm{x}} \mathrm{x}_{\mathrm{j}}\right)^{2}+\left(\mathrm{y}_{\mathrm{i}}-\mathrm{y}_{\mathrm{j}}\right)^{2}
$$

the fitness function calculates the values of both initial plant and new generated plant. If the fitness function is fulfilled, then there is no overlapping otherwise the new iteration will be performed until the overlapping will be removed.

\section{C.Termination Point}

In the problem of network coverage, the Plant Propagation Algorithm is executed till the whole intersection is not properly eliminated and the coverage of network is equal to maximum total area coverage.

\section{RESULT ANALYSIS}

The results of this algorithm is analysed by considering the total time that is required from the very initial state to final deployment of network sensor nodes. The given number of network sensors versus total time for computation is taken as a comparison to analyze which algorithm give fruitful results. The test is conducted with applied Algorithm i.e Plant Propagation Algorithm and Genetic Algorithm. 6 sensor nodes are taken that have random coordinates. The 
following figure shows the final output of PPA as well as the Generic Algorithm.

A. Initial Random Placement of nodes

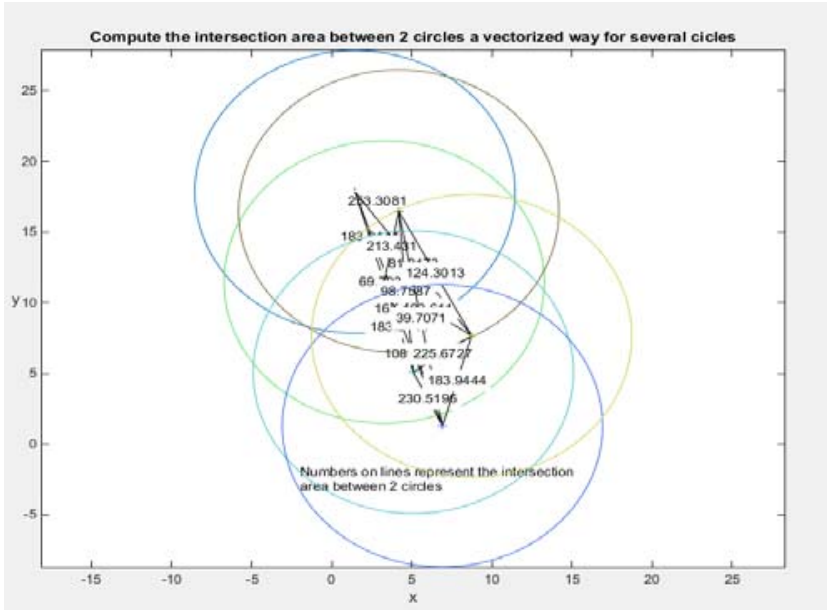

Fig.4. Initial Deployment

B. Final Placement of six network nodes with PPA.

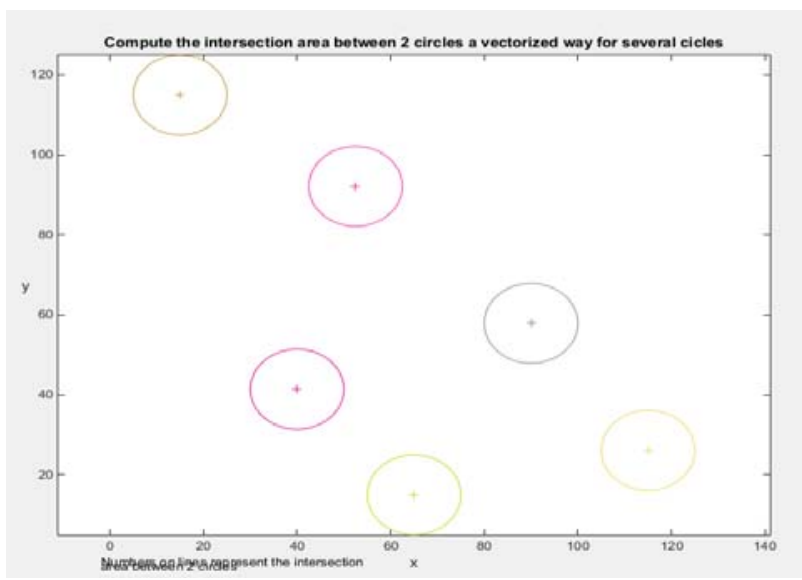

Fig.5. Final placement of nodes with Plant propagation Algorithm

C. Final Placement of six nodes with Genetic Algorithm

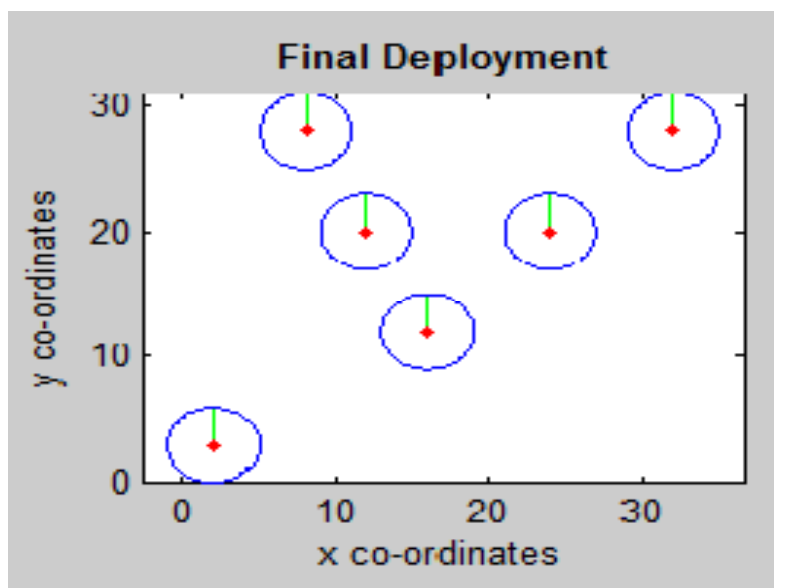

Fig.5 Final placement of six nodes with Genetic Algorithm
TABLE II. TIME COST VERSUS NUMBER OF NODES

\begin{tabular}{|c|c|c|}
\hline \multirow{2}{*}{$\begin{array}{c}\text { Number of } \\
\text { sensor nodes }\end{array}$} & \multicolumn{2}{|c|}{ Time cost( in seconds) } \\
\cline { 2 - 3 } & $\begin{array}{c}\text { Plant } \\
\text { Propagation } \\
\text { algorithm }\end{array}$ & $\begin{array}{c}\text { Genetic } \\
\text { algorithm }\end{array}$ \\
\hline 5 & 0.19 & 0.52 \\
\hline 10 & 1.23 & 1.56 \\
\hline 20 & 1.97 & 2.04 \\
\hline 30 & 2.85 & 3.06 \\
\hline 40 & 4.19 & 4.39 \\
\hline
\end{tabular}

The data represented in the above table is given in the form of bar chart and graph as following diagrams.

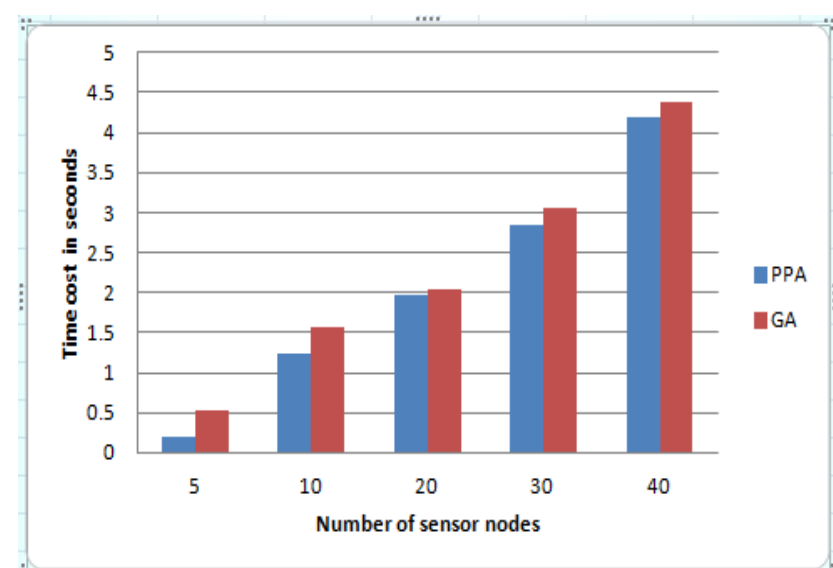

Fig. 6.Bar Graph of Total Time versus Number of sensors

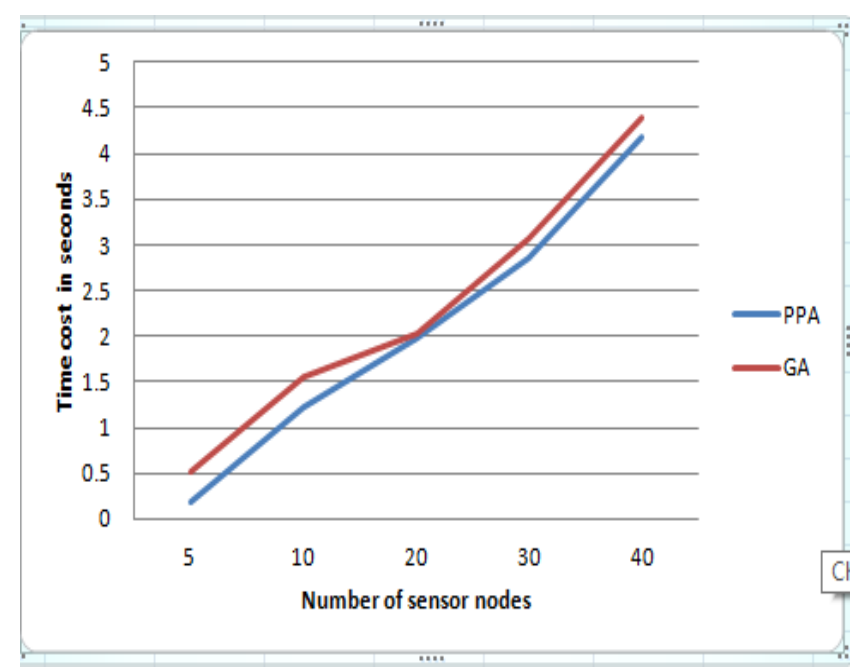

Fig. 10. Line Graph of Total Time versus Number of sensors

\section{CONCLUSION}

In this paper, it is concluded that the coverage in wireless sensor network has the great impact on quality of service of a network. Various techniques and method are reviewed that are used to overcome the problem of coverage. The coverage is heavily influenced by the deployment of nodes. The various issues founded regarding the deployment include homogeneous or heterogeneous deployment of 
sensor nodes and deterministic or random deployment of sensor nodes. Many paper focused on the random deployment of homogeneous sensor nodes to maximize the coverage area. In the analyses of outcomes of this algorithm, the placement of sensor nodes are represented. The final placement of sensor network nodes given by Plant Propagation and Genetic Algorithm is shown as a results. The very initial placement of network sensor nodes has maximum area which is intersected with each other. Both the given techniques are applied over the intersected area and it is found that the intersection is properly removed from the network. The outputs of both the techniques are compared by taking the parameters of total time cost versus number of network nodes. At the end it is concluded the results calculated by PPA are highly efficient than the genetic algorithm. It is also found that PPA decrease the time cost. The PPA gives maximum area coverage in the network with less time cost. The PPA only provides the coverage and time cost and did not concern with the parameters which are related to energy. In future PPA energy efficient area coverage network can be developed.

\section{REFERENCES}

[1] Raymond Mulligan, Habib M. Ammari," Coverage in Wireless Sensor Networks: A Survey", Research Lab Department of Computer Science, Hofstra University Hempstead, NY 11549, USA, Network Protocols and Algorithms, ISSN 1943-3581 2010, Vol. 2, No. 2.

[2] Anju Sangwan, Rishi Pal Singh," Survey on Coverage Problems in Wireless Sensor Networks", Published online: 30 September 2014 (C) Springer Science+Business Media New York 2014, Wireless Pers Commun (2015) 80:1475-1500,DOI 10.1007/s11277-014-2094-3.

[3] Moustafa A. Youssef, Adel Youssef, Mohamed F. Younis," Overlapping Multihop Clustering for Wireless Sensor Networks", IEEE TRANSACTIONS ON PARALLEL AND DISTRIBUTED SYSTEMS, VOL. 20, NO. 12, DECEMBER 2009.

[4] Muhammad Sulaiman, Abdellah Salhi, Birsen Irem Selamoglu, and Omar Bahaaldin Kirikchi "A Plant Propagation Algorithm for Constrained Engineering Optimisation Problems", Hindawi Publishing
Corporation Mathematical Problems in Engineering, Volume 2014, Article ID 627416, 10 pages ,http://dx.doi.org/10.1155/2014/627416

[5] Ravneet Kaur, Ashwani Kumar,"An Approach for Selecting Optimum Number of Base Stations and Optimizing Site Locations using Flower Pollination Algorithm", International Journal of Computer Applications (0975 - 8887) Volume 133 - No.10, January 2016.

[6] Shubhdeep Kaur, R S Uppal,” Dynamic Deployment of Homogeneous Sensor Nodes Using Genetic Algorithm with Maximum Coverage”, IEEE, 15109873, Computing for Sustainable Global Development (INDIACom), 2015 2nd International Conference on 11-13 March 2015, New Delhi, India.

[7] Sandeep Singh Syan, Amandeep Kaur Virk," Node Placement for Efficient Coverage in Homogeneous Wireless Sensor Networks", International Journal of Engineering Trends and Technology (IJETT) - Volume4 Issue 7- July 2013

[8] Prabhudutta Mohanty, Priyaranjan Mohapatra,"Maximum Coverage in WSN using Optimal Deployment Technique", IJCA Special Issue on "2nd National Conference- Computing, Communication and Sensor Network" CCSN, 2011

[9] GaoJun Fan, ShiYao Jin," Coverage Problem in Wireless Sensor Network: A Survey", JOURNAL OF NETWORKS, VOL. 5, NO. 9, SEPTEMBER 2010.

[10] Deying Li, Hai Liu," Sensor Coverage in Wireless Sensor Networks", School of Information, Renmin University of China, Department of Computer Science, Hong Kong Baptist University, Hong Kong. @inproceedings $\{$ Li2008SensorCI, Published 2008.

[11] S. Indhumathi, D. Venkatesan," Improving Coverage Deployment for Dynamic Nodes using Genetic Algorithm in Wireless Sensor Networks", Indian Journal of Science and Technology, Vol 8(16), DOI: 10.17485/ijst/2015/v8i16/62538, July 2015.

[12] Parul Agarwal, Shikha Mehta," Nature-Inspired Algorithms: State-of-Art, Problems and Prospects", International Journal of Computer Applications (0975 8887) Volume 100 - No.14, August 2014. 